\title{
The Growing Spectrum of Human Diseases Caused by Inherited CDC42 Mutations
}

\author{
Helen C. Su ${ }^{1} \cdot$ Jordan S. Orange ${ }^{2}$ \\ Published online: 16 May 2020 \\ (C) This is a U.S. Government work and not under copyright protection in the US; foreign copyright protection may apply 2020
}

\begin{abstract}
Several recent studies provide valuable new information that expands the spectrum of human disease associated with mutations in $C D C 42$.
\end{abstract}

CDC42, an intracellular member of the Rho-family GTPases, is a key regulator of cell polarity that is highly conserved among eukaryotes (reviewed in [1]). By regulating the assembly of actin cytoskeletal structures in a temporal-spatial manner, CDC42, along with RAC and RHO A, controls cell shape and cell movement. In this way, CDC42 plays an important role in migration and directed functions, processes that are integral for embryonic development, hematopoiesis, or immune system function. CDC42 also influences other functions such as cell growth and proliferation through effects on multiple downstream cell signaling pathways, via actin-based platforms and/or direct interactions of CDC42 with other effectors.

As a GTPase, CDC42 functions as an intracellular molecular switch. It cycles between two forms - an active form when bound to GTP and an inactive form when bound to GDP - subject to complex regulation by GTPase activation proteins (GAPs), guanosine exchange factors (GEFs), and guanosine dissociation inhibitors (GDIs). When activated, CDC42 undergoes a conformational change that enables it to associate with membranes and to interact with effector molecules, which in turn undergo their own conformational changes to exert downstream biochemical functions.

Helen C. Su

hsu@niaid.nih.gov

1 Laboratory of Clinical Immunology and Microbiology, Division of Intramural Research, National Institute of Allergy and Infectious

Diseases, National Institutes of Health, Bethesda, MD 20892, USA

2 Department of Pediatrics, Vagelos College of Physicians and Surgeons, New York-Presbyterian Morgan Stanley Children's Hospital, Columbia University, New York, NY 10032, USA
To understand how CDC42 biologically functions, much work had been done using cell lines that express dominantly negative or constitutively active mutants. However, these mutants can exert non-specific effects by binding guanine nucleotide exchange factors that not only affect CDC42 but also other Rho-family GTPases. Physiological studies using mice rendered genetically deficient in $\mathrm{Cdc} 42$ have been limited by embryonic lethality. However, mice rendered conditionally deficient for $\mathrm{Cdc} 42$ revealed its requirement for proper tissue morphogenesis and functioning, including effects on the cardiac, nervous, hematopoietic, and immune systems.

Clues provided by studies in mutant mice were soon followed by the identification of humans with inherited CDC42 mutations. From 2015 onward, the first patients were reported to have a de novo heterozygous missense mutation in CDC42 at p.Tyr64Cys, associated with a constellation of features termed as Takenouchi-Kosaki syndrome, which includes dysmorphism, developmental delay, macrothrombocytopenia, and in one case immunodeficiency [2-4]. In 2018, Martinelli and colleagues reported 15 more patients with de novo heterozygous missense mutations in CDC42, several of which were recurrent [5]. Disease characteristics shared by the patients included poor growth, facial dysmorphism, neurodevelopmental abnormalities, malformations of the heart, and non-specific hematological and immune aberrations.

More recently, widespread use of whole exome sequencing in patients with complex or severe conditions has led to discoveries that heterogeneous disease phenotypes can be associated with mutations in the same gene. Along this line, Lam and colleagues recently reported four patients with recurrent de novo heterozygous missense mutations in CDC42 at p.Arg 186Cys, a variant not previously associated with Takenouchi-Kosaki syndrome [6]. These patients had a different disease, termed as NOCARH (neonatal-onset cytopenia 
with dyshematopoiesis, autoinflammation, rash, and hemophagocytic lymphohistiocytosis (HLH)). The pathogenic hallmark of HLH is defective NK cell cytotoxicity in the context of other hyperactive immune responses. Indeed, defects in NK cell cytotoxicity, migration, and conjugate formation, as well as overproduction of proinflammatory cytokines, were all observed in NOCARH. Importantly, these observations help to explain why most of the patients showed some improvement after treatment with IL-1 and/or IFN- $\gamma$ antagonists. Furthermore, they explain why HSCT, which is the treatment of choice for HLH, cured a disease in one patient who underwent this procedure.

Additionally, similar clinical findings were reported in four other patients who had a severe neonatal autoinflammatory disease, including HLH/macrophage activation syndrome. Gernez and colleagues found similar de novo heterozygous missense mutations nearby in the $\mathrm{C}$-terminal region of $C D C 42$ and observed that these patients also improved after treatment with IL-1 receptor antagonists [7].

More recently, including in this and an earlier issue of the Journal of Clinical Immunology, five reports add to the rapidly expanding body of knowledge about this fascinating new disease. The first, by Bucciol and colleagues, describes the oldest patient so far reported, who at 26 years was found to have Takenouchi-Kosaki syndrome with the usual p.Tyr64Cys mutation in a conserved domain of $C D C 42$ required for its GTPase activity [8]. Surprisingly, the patient also had systemic autoinflammation previously associated with NOCARH and other C-terminal region mutations, as well as recurrent severe infections leading to bronchiectasis and death from sepsis. Several new features were observed including myelofibrosis, pulmonary hemorrhage, and vascular malformations. The second, by He and Yuan, described a patient with the p.Arg186Cys mutation, dysmorphism, and NOCARH along with elevated serum IgE and a possible mild protein allergy [9]. The third, by Bekhouhe and colleagues, described another patient with the p.Arg186Cys mutation and similar findings including elevated serum IgE, but who continued to have difficulties despite HSCT or IL-1 antagonists [10]. Thus, these cases potentially add to the phenotypic spectrum but open questions regarding genotype-phenotype correlations of CDC42 as well as the potential for other clinical or secondary influences.

The fourth report, by Verboon and colleagues, describes two additional siblings with a shared heterozygous p.Arg186Cys mutation, inherited via paternal germline mosaicism [11]. Both patients presented with congenital pancytopenia and myelofibrosis, with one dying of complications after HSCT and the other dying after progressing to acute myeloid leukemia in infancy. This distinct phenotype, and the demonstration that the mutation exerts deleterious effects on the function of hematopoietic stem and progenitor cells, led the authors to explore the possibility of $\mathrm{CDC} 42$ dysfunction more broadly. Intriguingly, they saw a correlation with decreased CDC42 expression in the bone marrow in other cases of primary myelofibrosis, suggesting the importance of this common pathway in disease pathogenesis. Finally, a fifth report recently described a child with a novel de novo heterozygous p.Cys81Tyr mutation, who had syndromic immunodeficiency, autoinflammation, HLH, and Hodgkin's lymphoma, illustrating again that the broad phenotypic spectrum of this disease includes malignancy [12].

Together, these five recent studies provide valuable new information that expands the spectrum of human disease associated with mutations in $C D C 42$. However, they also raise other interesting questions: As more patients are identified, can they shed new light into genotype-phenotype relationships? If so, what are the specific molecular perturbations that can explain how different mutations give rise to the different clinical presentations? At present, most of the severe immunologic syndrome presentations stem predominantly from variation at residue 186 . There is strong biochemical basis for how this variation impacts CDC42 biology as the variant is abnormally palmitoylated, which causes inappropriate CDC42 localization with protein retention at the Golgi complex. However, more detailed studies of the other variants are needed to elucidate how they lead to an immunological/ autoinflammatory disease and why some patients with the same variants might fail to do so. Finally, do either germline or somatic mutations in CDC42 underlie some forms of myelofibrosis or leukemia, and can modulating CDC42 activity be used therapeutically for these conditions? Clearly, there is much more to be learned from patients about how CDC42 functions physiologically in humans.

Acknowledgments We thank Dr. Michael Lam for helpful suggestions. HCS is supported by the Intramural Research Program, NIAID, NIH, and JSO by NIAID grant RO1-AI120989.

\section{References}

1. Melendez J, Grogg M, Zheng Y. Signaling role of Cdc42 in regulating mammalian physiology. J Biol Chem. 2011;286(4):2375-81.

2. Takenouchi T, Kosaki R, Niizuma T, Hata K, Kosaki K. Macrothrombocytopenia and developmental delay with a de novo CDC42 mutation: yet another locus for thrombocytopenia and developmental delay. Am J Med Genet A. 2015;167A(11):2822-5.

3. Takenouchi T, Okamoto N, Ida S, Uehara T, Kosaki K. Further evidence of a mutation in $\mathrm{CDC} 42$ as a cause of a recognizable syndromic form of thrombocytopenia. Am J Med Genet A. 2016;170A(4):852-5.

4. Motokawa M, Watanabe S, Nakatomi A, Kondoh T, Matsumoto T, Morifuji K, et al. A hot-spot mutation in CDC42 (p.Tyr64Cys) and novel phenotypes in the third patient with Takenouchi-Kosaki syndrome. J Hum Genet. 2018;63(3):387-90.

5. Martinelli S, Krumbach OHF, Pantaleoni F, Coppola S, Amin E, Pannone L, et al. Functional Dysregulation of CDC42 causes 
diverse developmental phenotypes. Am J Hum Genet. 2018;102(2): 309-20.

6. Lam MT, Coppola S, Krumbach OHF, Prencipe G, Insalaco A, Cifaldi $\mathrm{C}$, et al. A novel disorder involving dyshematopoiesis, inflammation, and HLH due to aberrant CDC42 function. J Exp Med. 2019;216(12):2778-99.

7. Gernez Y, de Jesus AA, Alsaleem H, Macaubas C, Roy A, Lovell $\mathrm{D}$, et al. Severe autoinflammation in 4 patients with C-terminal variants in cell division control protein 42 homolog (CDC42) successfully treated with IL-1beta inhibition. J Allergy Clin Immunol. 2019;144(4):1122-5 e6.

8. Bucciol G, Pillay B, Casas-Martin J, Delafontaine S, Proesmans M, Lorent N, et al. Systemic inflammation and myelofibrosis in a patient with Takenouchi-Kosaki syndrome due to CDC42 Tyr64Cys mutation. J Clin Immunol. 2020.

9. He T, Huang Y, Ling J, Yang J. A New Patient with NOCARH Syndrome Due to CDC42 Defect. J Clin Immunol. 2020;40(4). https://doi.org/10.1007/s10875-020-00786-7.
10. Bekhouche B, Tourville A, Ravichandran Y, Tacine R, Abrami L, Dussiot M, et al. A toxic palmitoylation of Cdc42 enhances NFkappaB signaling and drives a severe autoinflammatory syndrome. J Allergy Clin Immunol. 2020.

11. Verboon JM, Mahmut D, Kim AR, Nakamura M, Abdulhay NJ, Nandakumar SK, et al. Infantile Myelofibrosis and Myeloproliferation with CDC42 Dysfunction. J Clin Immunol. 2020;40(4). https://doi.org/10.1007/s10875-020-00778-7.

12. Szczawinska-Poplonyk A, Ploski R, Bernatowska E, Pac M. A novel CDC42 mutation in an 11-year old child manifesting as syndromic immunodeficiency, autoinflammation, hemophagocytic lymphohistiocytosis, and malignancy: a case report. Front Immunol. 2020;11:318.

Publisher's Note Springer Nature remains neutral with regard to jurisdictional claims in published maps and institutional affiliations. 